\title{
ADVANCES AND APPLICATIONS IN LOGIC
}

\section{CHARLES DORSETT}

Department of Mathematics

Texas A\&M University-Commerce

Texas 75429

USA

e-mail: charles.dorsett@tamuc.edu

\begin{abstract}
Within a recent paper a long overlooked reality in logic was presented and additions and changes in the previous calculus of logic were given. In this paper the new expanded calculus of logic is further investigated.
\end{abstract}

\section{Introduction and Preliminaries}

Logic is the foundation upon which mathematics and other fields of study have been built. However recent discoveries in the study of foundational topology have revealed a long overlooked, meaningful reality in the study of logic that required additions and changes; namely, the existence of topological properties $P$ for which "not- $P$ " does not exist [1].

In logic, a sentence which is either true or false, but not both, is called a proposition [3]. The negation of a proposition $P$, which in this paper is denoted by "not- $P$ ", is the proposition not $P$ [3]. In past studies of logic, 
no discussion or restrictions were given for "not- $P$ " and truth values were assigned; if $P$ is true, then "not- $P$ " is false and if $P$ is false, "not- $P$ " is true; and "not- $P$ ", with those truth values, was used in the calculus of logic. However, as given above, there are propositions $P$ for which "not- $P$ " does not exist and the assignment of truth values for "not- $P$ " above would be incorrect, totally overlooking the possibility that "not- $P$ " does not exist; creating the real possibility of false conclusions. Thus care must be used to determine whether or not "not- $P$ " exists for a proposition $P$ and, in the case "not- $P$ " does not exist, assigning "not- $P$ " only truth value "false".

As long known in the study of logic, there are propositions with only truth value "true". With the addition of propositions that do not exist, propositions can be broken up into three distinct categories given below.

Definition 1.1. Let $P$ be a proposition. If $P$ has truth values both "true" and "false", then $P$ is said to be non-identity existent. If $P$ has only truth value "true", then $P$ is said to be an identity. If $P$ has only truth value "false", then $P$ is said to be non-existent.

If $P$ and $Q$ are identities, then, by use of a truth table, $P$ and $Q$ are logically equivalent written as $P=Q$. Below $L$ will be used to represent those propositions that are identities. Likewise, if $P$ and $Q$ are non-existent, then by use of a truth table, $P$ and $Q$ are logically equivalent written as $P=Q$. Below $N E$ will be used to represent those propositions that are non-existent.

\section{A Least and Strongest Proposition}

Theorem 2.1. Let $P$ be non-identity existent for which "not-P" is non-existent. Then $(P$ or "not-P") $=P$. 
The proof is straightforward using a truth table and is omitted.

In classical calculus of logic, $(P$ or "not- $P$ " $)$ is an identity. As established later in this paper, there are non-identity existent propositions $P$ for which "not- $P$ " is non-existent and, thus, the addition of non-existent propositions created a required change in the calculus of logic and care must be taken.

Theorem 2.2. Let $P$ be a proposition for which both $P$ and "not-P" are non-identity existent, or $P$ is non-existent, or $P$ is an identity. Then ( $P$ or "not- $P$ ") is an identity.

The proof is straightforward and omitted.

Thus, there are many different representations of an identity proposition.

Theorem 2.3. Let $P$ be a proposition. Then $(P$ and $L)=P$ and $L$ is the identity element in $\mathcal{P}=\{Q: Q$ is a proposition $\}$.

Proof. There are three cases to consider; (1) $P$ is non-identity existent, (2) $P$ is non-existent, and (3) $P=L$. In all three cases, use of a truth table gives $(P$ and $L)=P$, and thus $L$ is an identity element in $\mathcal{P}$. If $Z$ is an identity element in $\mathcal{P}$, then $(L$ and $Z)=L,(L$ and $Z)=Z$, and $L=Z$. Thus $L$ is the identity element in $\mathcal{P}$.

Below $\mathcal{P}$ will be used as given in Theorem 2.3.

Theorem 2.4. Let $P$ be a proposition. Then $P$ implies $L$.

As above, there are three cases to consider; $P$ is non-identity existent, $P$ is non-existent, and $P$ is an identity. Then truth tables can be easily used to show that in each case $(P$ implies $L)$ is a tautology. Thus $L$ is the least element of $\mathcal{P}$. 
Corollary 2.1. $L$ is the least element and the identity element in $\mathcal{P}$.

Corollary 2.2. If $\mathcal{U}$ is a subset of $\mathcal{P}$ containing $L$, then $\mathcal{U}$ has least element $L$ that is the identity element.

Thus, the addition of non-existent propositions has revealed new, important properties in the calculus of logic.

Theorem 2.5. Let $P$ be a proposition. Then (NE implies $P$ ) and $N E$ is the strongest element of $\mathcal{P}$.

As above, there are the three cases to consider and in all three cases, truth tables show $(N E$ implies $P$ ) is a tautology. Thus $N E$ is the strongest element in $\mathcal{P}$.

Corollary 2.3. If $\mathcal{U}$ is a subset of $\mathcal{P}$ containing $N E$, then $\mathcal{U}$ has strongest element $N E$.

Corollary 2.4. If $\mathcal{U}$ is a subset of $\mathcal{P}$ containing $L$ and $N E$, then $\mathcal{U}$ contains strongest element $N E$ and least identity element $L$.

Thus additional important properties in the calculus of logic are revealed.

Theorem 2.6. Let $P$ be a proposition such that both $P$ and "not-P" are non-identity existent or $P=N E$ or $P=L$. Then ( $P$ and "not-P") is non-existent.

In all three cases truth tables show $(P$ and "not- $P$ " $)=N E$ is a tautology.

Thus $N E$ has many representations.

Theorem 2.7. Let $P$ be a proposition such that "not-P" is nonidentity existent. Then each of $P$ and "not-("not-P")" are non-identity existent and $P=$ "not-("not-P")". 
Proof. The proposition $P$ is exactly one of non-identity existent, $N E$, and $L$. If $P=N E$, then "not- $P$ " $=L$, which is a contradiction, and if $P=L$, then "not- $P$ " $=N E$, which is a contradiction. Thus $P$ is nonidentity existent. Then, if $P$ is assigned truth values "true" then "false", "not- $P$ " would be assigned "false" then "true", respectively, and "not("not-P")" would be assigned "true" then "false", respectively, which is $P$.

Corollary 2.5. Let $P$ be a proposition for which "not-P" is nonidentity existent or $P$ is non-existent or $P$ is an identity. Then ( $P$ or "not-P") is an identity.

Theorem 2.8. Let $P$ be a proposition. Then "not-("not-P")" is nonidentity existent iff "not-P" is non-identity existent and $P=$ "not(“not-P")".

Proof. Suppose "not-("not- $P$ ")" is non-identity existent. Let $Q=$ "not- $P$ ". Then "not- $Q$ " = "not-("not- $P$ ")", which is non-identity existent and, by Theorem 2.7, $Q=$ "not- $P$ " is non-identity existent. Then, by Theorem 2.7, $P$ is non-identity existent and $P=$ "not-("not- $P$ ")".

Conversely suppose "not- $P$ " is non-identity existent and $P=$ "not("not-P")". Then by Theorem 2.7, $P$ is non-identity existent. Hence "not-("not-P")" is non-identity existent.

Corollary 2.6. Let $P$ be a proposition such that "not-("not-P")" is non-identity existent. Then "not-P" = "not-("not-("not-P")")", which is non-identity existent.

Theorem 2.9. Let $\mathcal{U}=\{P: P$ is a proposition and "not-("not-P")" is non-identity existent $\}$. Then $\mathcal{U}$ has no strongest element and no least element. 
Proof. Let $P \in \mathcal{U}$. Then, by results above, $P \in \mathcal{U}$ and "not-P" $\in \mathcal{U}$. Suppose $\mathcal{U}$ has strongest element $S$. Then "not- $S$ " $\in \mathcal{U}$ and $(S$ implies "not-S"). Then (( $S$ implies $S)$ and $(S$ implies "not-S")), but, if $S$ is true, then "not- $S$ " is false, $(S$ implies $S)$ is true, $(S$ implies "not- $S$ ") is false, and ( $S$ implies $S)$ and $(S$ implies "not- $S "))$ is false. Hence $\mathcal{U}$ has no strongest element.

Suppose $\mathcal{U}$ has least element $W$. Then ("not- $W$ " implies $W$ ) and ("not- $W$ " implies "not- $W$ "), and, in a similar manner to that above, $\mathcal{U}$ has no least element.

Theorem 2.10. Let $P$ be a proposition. Then $P=N E$ iff "not-("not$P ") "=N E$.

Proof. Clearly, if $P=N E$, then "not-("not- $P$ ")" $=N E$. Thus consider the case that "not-("not- $P$ ")" $=N E$. Then "not- $P$ " is nonidentity existent, or $N E$, or $L$. By Theorem 2.7, "not- $P$ " is not nonidentity existent and clearly "not- $P$ " is not $N E$. Thus "not- $P$ " $=L$. Then $P \neq L$. Suppose $P \neq N E$. Then $P$ is non-identity existent, $(P$ and "not $-P ")=P$, and $(P$ or "not- $P$ " $)=L$. Thus $P=(P$ and "not- $P$ " $)=$ $\operatorname{not}-(P$ or $"$ not $-P ")=$ "not- $L "=N E$, which is a contradiction. Thus $P=N E$.

Theorem 2.11. If $P=L$, then "not-("not-P")" $=P$.

The proof is straightforward and omitted.

The work above raise questions about the converses of Theorems 2.7 and 2.11. Below additional insights into the new calculus of logic are given and applied to resolve the questions concerning converses. 


\section{Additional Insights and Resolution of the Questions}

Within logic and its application "and", "or", and "implications" have an important role. In the continued study of foundational topology [2], a connection between "and" and "implication" was discovered: If $P$ and $Q$ are non-identity existent propositions, then $(P$ implies $Q)$ iff $((P$ and $Q)=P)$. Since, from above, $N E$ is the strongest proposition, for each proposition $Q,(N E$ implies $Q)$ and $((N E$ and $Q)=N E)$; and since $L$ is the least proposition and the identity element, for each proposition $P,(P$ implies $L)$ and $\quad((P$ and $L)=P)$. Thus questions about a connection between "or" and "implication" arise.

Theorem 3.1. If $P$ and $Q$ are non-identity existent, then $(P$ implies $Q)$ iff $(P$ or $Q)=Q$, and for each proposition $W,(N E$ implies $W),(N E$ or $W)=W,(W$ implies $L)$, and $(W$ or $L)=L$.

Proof. Since $P$ and $Q$ are non-identity existent, then, by use of a classical truth table, $(P$ implies $Q)$ iff $(P$ or $Q)=Q$. For a proposition $W$, there are three cases to consider and in all three cases, use of a truth table establishes ( $N E$ implies $W$ ) as a tautology and, thus always true. In the same manner each of the following are tautologies and always true.

Definition 3.1. Let $P$ and $Q$ be propositions. Then $P$ is stronger than $Q$ iff $(P$ implies $Q)$ and $(P \neq Q)$, where $(P \neq Q)$ iff when $P$ has truth value "true", $Q$ has truth value "false" and when $P$ has truth value "false", $Q$ has truth value "true".

Theorem 3.2. Let $P$ and $Q$ be propositions such that each of "not-P" and $Q$ are non-identity existent. Then ( $P$ is stronger than $Q$ ) iff $(Q$ and "not-P") is non-identity existent. 
Proof. Since "not- $P$ " is non-identity existent, then, by the results above, $P$ is non-identity existent. Use of the given conditions in a truth table shows $(Q$ and "not- $P$ ") is non-identity existent and ( $P$ is stronger than $Q)$ iff ( $Q$ and "not- $P$ ") is non-identity existent.

Thus propositions $P$ and $Q$ satisfying the conditions in Theorem 3.2 can be used to give an additional non-identity existent proposition.

Theorem 3.3. Let $P$ and $Q$ be propositions such that both "not-P" and "not-Q" are non-identity existent. Then ( $P$ is stronger than $Q)$ iff ( $P$ and "not-Q") is non-existent.

Proof. Since each of "not- $P$ " and "not-Q" are non-identity existent, then both $P$ and $Q$ are non-identity existent and, by the results above, ( $P$ is stronger than or equal to $Q)$ iff $(P$ and $Q)=P$. Thus $(P$ is stronger than or equal to $Q)$ iff ( $P$ and "not- $Q$ ") $=(P$ and $Q)$ and "not- $Q$ " $=P$ and $(Q$ and "not- $Q ")=(P$ and $N E)=N E$.

Thus propositions $P$ and $Q$ satisfying the conditions in Theorem 3.3 can be used to generate a non-existent proposition.

Theorem 3.4. Let $P$ and "not-Q" be non-identity existent. Then (P or "not-Q") is non-identity existent.

Proof. Since "not- $Q$ " is non-identity existent, then $Q$ is non-identity existent and use of a truth table shows ( $P$ and "not- $Q$ ") is non-identity existent.

Below, the results above are used to show the converses of Theorems 2.7 and 2.11 are false. 
Example 3.1. Let $P$ and $Q$ be propositions such that both "not- $P$ " and "not- $Q$ " are non-identification and existent and ( $P$ is stronger than $Q$ ), and let $W=(Q$ or "not- $P$ " $)$. Then $W$ is non-identity existent and "not- $W$ " $=(P$ and "not- $Q ")=N E$. Then $W$ is non-identity existent and "not- $W "=L \neq W$, and the converse of Theorem 3.11 is false.

\section{References}

[1] C. Dorsett, An overlooked reality in logic requiring additions, accepted by Universal Journal of Mathematics and Mathematical Sciences.

[2] C. Dorsett, No between topological properties for consecutive classical separation axioms, Fundamental Journal of Mathematics and Mathematical Sciences 13(1) (2020), 1-14.

[3] R. Johnsonbaugh, Discrete Mathematics, Prentice Hall, 2001. 